\title{
Politique publique et développement des chaînes alimentaires courtes de proximité : une analyse des PDR de la Région Occitanie
}

Public policy and the development of short local chains: an analysis of the RDPs in the Occitanie Region

Fredéric Wallet et Melise Dantas Machado Bouroullec

\section{OpenEdition \\ Journals}

\section{Édition électronique}

URL : https://journals.openedition.org/developpementdurable/18989

DOI : 10.4000/developpementdurable.18989

ISSN : 1772-9971

Éditeur

Association DD\&T

Référence électronique

Fredéric Wallet et Melise Dantas Machado Bouroullec, «Politique publique et développement des chaînes alimentaires courtes de proximité : une analyse des PDR de la Région Occitanie »,

Développement durable et territoires [En ligne], Vol. 12, $n^{\circ} 1$ | Mai 2021, mis en ligne le 26 mai 2021, consulté le 28 mai 2021. URL : http://journals.openedition.org/developpementdurable/18989; DOI : https://doi.org/10.4000/developpementdurable.18989

Ce document a été généré automatiquement le 28 mai 2021.

\section{(c) (1) \&}

Développement Durable et Territoires est mis à disposition selon les termes de la licence Creative Commons Attribution - Pas d'Utilisation Commerciale 4.0 International. 


\section{Politique publique et développement des chaînes alimentaires courtes de proximité : une analyse des PDR de la Région Occitanie}

Public policy and the development of short local chains: an analysis of the RDPS in the Occitanie Region

Fredéric Wallet et Melise Dantas Machado Bouroullec

Cet article a bénéficié du support de la Région Occitanie dans le cadre du financement du projet Circuits alimentaires de proximité - les Plateformes d'approvisionnement territorialisées (Cappap). Par ailleurs, les auteurs tiennent à remercier l'équipe de l'ODR pour son aide précieuse à l'accès et au traitement des données des PDR.

1 En Europe, les chaînes alimentaires courtes de proximité (CACP) sont devenues un élément central des stratégies de valorisation territoriale (Jouen et Lorenzi, 2014). Le dynamisme des initiatives est tangible sur de nombreux territoires, laissant apparaître des perspectives encourageantes, mais aussi des points de fragilité. Nous adoptons dans cet article le terme générique CACP dans le sens de la définition adoptée par la Commission européenne. Ainsi, les CACP sont des «circuits d'approvisionnement impliquant un nombre limité d'opérateurs économiques engagés dans la coopération, le développement économique local et des relations géographiques et sociales étroites entre les producteurs, les transformateurs et les consommateurs » (comité des régions, 2011, révisé par le Parlement européen, 2013). Cela intègre les circuits courts avec aucun ou un intermédiaire (Chaffotte et Chiffoleau, 2007), ainsi que les circuits de proximité qui tiennent compte de la distance géographique et organisée entre les acteurs du système alimentaire (Praly et al., 2014). 
2 Depuis une dizaine d'années, des mesures dédiées aux CACP ont été progressivement structurées ou infléchies dans les dispositifs existants (Hérault-Fournier et PringentSimonin, 2012). Les autorités publiques, à différentes échelles, se sont emparées de la thématique pour répondre à des enjeux agricoles, alimentaires et de développement durable. Face à ces nouveaux enjeux, les Régions - nouvelles autorités de gestion de fonds européens - ont dû adapter leurs pratiques pour intégrer des formes nouvelles d'activités agricoles qui n'entraient pas dans les schémas traditionnels et appelaient des modalités de soutien appropriées. Cependant, la littérature consacrée aux politiques alimentaires territoriales se concentre presque exclusivement sur les démarches infrarégionales, voire, pour l'essentiel, métropolitaines (Brand et al., 2017; Moragues-Faus et Morgan, 2015). L'examen des stratégies régionales reste peu traité, soulignant à la fois le déficit d'outillage analytique pour rendre compte de cette évolution et le manque de données pratiques. La lisibilité des logiques d'intervention fait en effet défaut, tant en ce qui concerne les types de bénéficiaires, les formes d'interventions ou encore les critères de mise en application.

3 En France, la Région Occitanie ne fait pas exception. Espace régional marqué par le dynamisme et la variété des initiatives en CACP, la région combine, pour la programmation 2014-2020, deux PDR, Midi-Pyrénées et Languedoc-Roussillon. Ils s'appliquent à une configuration spatiale polymorphe articulant deux métropoles en forte croissance, une zone sous influence littorale et des espaces ruraux plus traditionnels, ainsi qu'une grande variété de systèmes de production agricoles.

4 L'objectif de l'article est de mieux comprendre comment les Régions ont souhaité soutenir le développement des CACP 2014-2020. Pour ce faire, les première et deuxième parties de l'article proposent un tour d'horizon du processus de structuration des dispositifs d'intervention publique en faveur des CACP, et une présentation de leur place dans la politique européenne de développement rural 2014-2020. La troisième partie présente le cadre d'analyse adopté pour investiguer les formes d'intervention publique en faveur des CACP. La quatrième rend compte de la diversité de stratégies et du poids plus effectif de la prise en compte des CACP dans les appels à projets. Enfin, nous discutons les perspectives en vue de la PAC post-2020 et de la configuration institutionnelle derrière les nouvelles politiques de développement régional en France.

\section{La structuration progressive des dispositifs d'intervention publique en faveur des CACP en France}

Selon Kneafsey et al. (2013), à la fin des années 2000, la France émerge comme un des pays européens les plus actifs dans l'appui institutionnel aux modes de vente rapprochant producteur et consommateur. Au niveau des politiques nationales, le Programme national nutrition santé (PNNS) lancé en 2001 a, dès sa seconde génération, œuvré en faveur du développement des productions agricoles en CACP, notamment en facilitant l'accès aux produits issus de ces circuits pour la restauration collective publique. 


\subsection{Le PNNS2 : stade initial de l'institutionnalisation des CACP dans la politique agricole}

6 En 2008, le Plan national d'action pour une politique de l'offre alimentaire sûre, diversifiée et durable (PNOA) charge les Directions régionales de l'alimentation, de l'agriculture et de la forêt (DRAAF), de l'élaboration des plans d'action régionaux (cf. figure 1). Une des 13 actions développées vise à recréer un lien entre producteurs et consommateurs. La promotion des CACP pour favoriser le lien entre les exploitants agricoles et les consommateurs (cueillette à la ferme, vente à la ferme, etc.) ou bien des structures collectives tels les magasins de producteurs et les Amap commence à être présente dans plusieurs programmes alimentaires français.

Figure 1. Un repère des programmes nationaux de soutien aux CACP

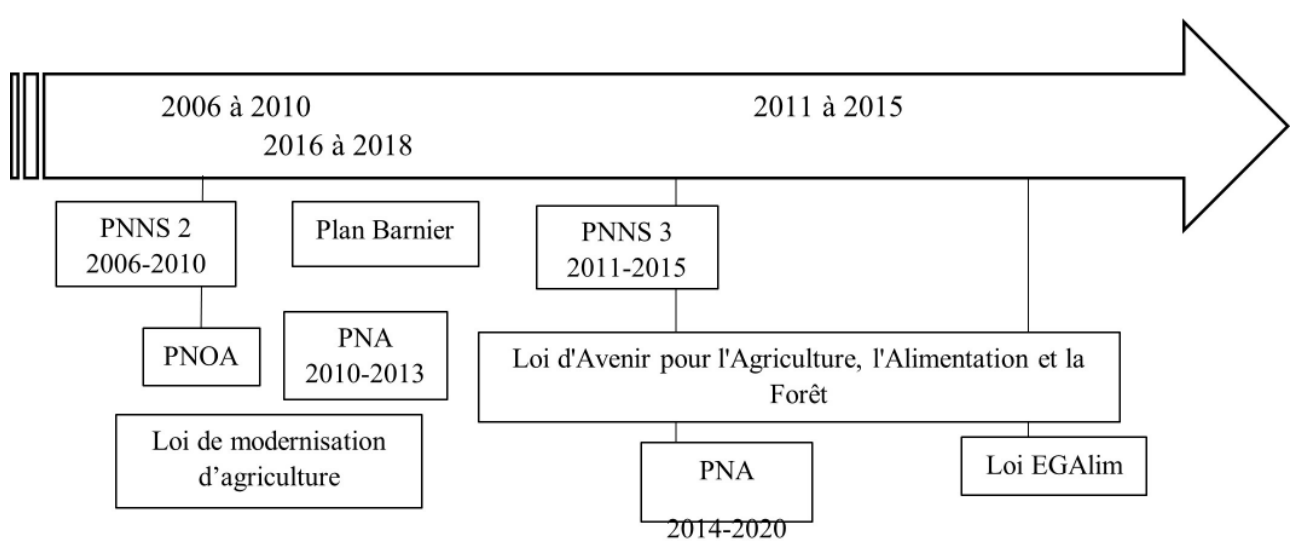

7 L'année 2010 marque un jalon important de la prise en compte des CACP, tout un plan d'action étant consacré à leur développement. Le plan Barnier cherche à améliorer et diffuser les connaissances sur les circuits courts; adapter la formation des agriculteurs aux exigences de ce mode de commercialisation; favoriser l'installation d'agriculteurs en circuits courts; et améliorer l'organisation des circuits courts. À ce stade, la définition des circuits courts insiste volontairement sur la réduction du nombre d'intermédiaires ( 0 ou 1$)$, sans trancher sur le débat complexe des frontières en matière de proximité géographique.

8 La loi de Modernisation de l'agriculture et de la pêche renforce l'objectif de l'État d'approvisionner les services de restauration collective avec des produits issus des CACP, qui impliquent une exploitation agricole ou une organisation collective regroupant plusieurs exploitations. Le but de l'État est «l'introduction des produits saisonniers, à faible impact environnemental eu égard à leurs conditions de production et de distribution, sous signe d'identification de la qualité et de l'origine, ou issus d'exploitations engagées dans une démarche de certification environnementale à hauteur minimale de $15 \%$ des commandes en 2010 et $20 \%$ en $2012^{1} »$. Elle prévoit également la mise en place d'une politique de l'alimentation à travers un Programme national pour l'alimentation (PNA), en liaison avec le PNNS. Pour favoriser l'approvisionnement de la restauration collective via les CACP, le code des marchés publics a ainsi été modifié par décret le 25 août 2011, donnant la possibilité aux acheteurs de " retenir parmi les critères de choix de l'offre économiquement la plus avantageuse les performances en matière de développement direct de produits de l'agriculture ». À plusieurs reprises, le seuil de dispense de publicité 
et de mise en concurrence a été relevé. Depuis 2020, il est passé de $25000 €$ HT à $40000 € \mathrm{HT}^{2}$ et renforce les achats de gré à gré.

\subsection{Le tournant des Projets alimentaires territoriaux}

9 Les CACP sont encore encouragés dans la loi d'Avenir pour l'agriculture, l'alimentation et la forêt de 2014 et son $\mathrm{PNA}^{3}$, qui soutient tout particulièrement l'émergence et la mise en œuvre des projets alimentaires territoriaux (PAT). Ce nouvel instrument est porteur d'une inflexion supplémentaire quant à l'affirmation de la reterritorialisation de l'agriculture, privilégiant plus explicitement le caractère local des CACP. Les PAT permettent de rapprocher les producteurs, les transformateurs, les distributeurs, les collectivités territoriales et les consommateurs pour développer l'agriculture locale et promouvoir une alimentation de qualité (Maréchal et al., 2018). Encore une fois, on glisse des circuits courts ( 0 ou 1 intermédiaire) aux circuits de proximité, mais sans aller jusqu'à normer cette dernière.

10 Plus récemment, les états généraux de l'alimentation ont conduit à la mise en place de la loi pour l'Équilibre des relations commerciales dans le secteur agricole et alimentaire et une alimentation saine, durable et accessible à tous ${ }^{4}$ (EGAlim). Parmi les mesures phares, l'article L. 230-5-1 prévoit qu'au plus tard en janvier 2022, au moins $50 \%$ de produits acquis par la restauration collective publique, dont $20 \%$ issus de l'agriculture biologique (AB), prennent en compte les coûts imputés aux externalités environnementales liées au produit pendant son cycle de vie. Les produits bénéficiant d'un signe ou mentions destinées à favoriser la qualité des produits tels «produit de montagne ", "fermier ", "produit à la ferme", "issu d'une exploitation de haute valeur environnementale», pourront bénéficier de ce nouveau marché. Cette progressive reconnaissance institutionnelle des CACP en France trouve un écho à travers le mouvement similaire qui se déploie dans le cadre de la Politique agricole commune.

\section{Un socle commun de mise en œuvre de la politique européenne de développement rural 2014-2020}

11 La politique européenne de développement rural vise à aider les régions de l'UE à relever les nombreux défis économiques, environnementaux et sociaux liés aux espaces ruraux du xxI ${ }^{e}$ siècle. Comme indiqué par Guérin (2006), quatre conceptions successives des politiques de développement rural se sont développées au fil du temps. Elles sont passées progressivement, post-1945, d'une vision agro-centrée basée sur les objectifs quantitatifs à des conceptions plus territorialisées, et répondant aux préoccupations de développement durable. Parallèlement, les changements de styles d'intervention publique sont schématiquement caractérisés par le passage d'une vision tutélaire de l'État à une conception plus partenariale selon laquelle l'État est un contributeur parmi d'autres. Actuellement entendue comme le second pilier de la PAC, la politique européenne de développement rural complète, à travers le Fonds européen agricole de développement rural (FEADER), le régime de paiements directs aux agriculteurs et les mesures permettant de gérer les marchés agricoles (premier pilier). 


\subsection{La programmation 2014-2020 : un cadre renouvelé de la politique de développement rural en Europe}

12 Pour la période 2014-2020, 20 pays membres ont opté pour un PDR national unique et 8 autres, y compris la France, pour un ou plusieurs programmes régionaux. Dans ce contexte de décentralisation, les États membres et les régions élaborent des PDR fondés sur les besoins de leurs territoires et portant sur, au moins, quatre des six priorités communes, inscrites dans le Règlement de développement rural (RDR3) : (1) favoriser le transfert de connaissances et l'innovation ; (2) améliorer la viabilité des exploitations agricoles et la compétitivité de tous les types d'agriculture; (3) promouvoir l'organisation de la chaîne alimentaire; (4) restaurer, préserver et renforcer la biodiversité; (5) promouvoir l'utilisation efficace des ressources et soutenir la transition vers une économie à faibles émissions de $\mathrm{CO}_{2}$ et résiliente aux changements climatiques; (6) promouvoir l'inclusion sociale, la réduction de la pauvreté et le développement économique. Les priorités du RDR3 se décomposent en 20 mesures de la programmation Feader qui peuvent être déclinées en sous-mesures, qui sont ellesmêmes déclinées en un ou plusieurs types d'opérations (Parlement européen et Conseil, 2013).

\subsection{Les CACP dans la politique de développement rural européenne}

13 Depuis les années 2000, les CACP sont soutenus à travers la politique européenne de développement rural dont le programme Liaison entre actions de l'économie rurale (Leader), un des axes du Feader. En 2010, le Comité des régions de l'UE a rendu un avis sur l'identification des mesures à mettre en œuvre pour soutenir les productions de terroir destinées à être commercialisées par des CACP (Jouen et Lorenzi, 2014). En 2011, ce comité émet des recommandations politiques pour les futures lignes directrices stratégiques que la Commission européenne pourrait suggérer aux États membres. Le développement des $\mathrm{CACP}$ est préconisé dans le cadre de la stratégie de développement rural (Comité des Régions, 2011) que nous cherchons ici investiguer. Selon Jouen et Lorenzi (2014), cette inflexion par rapport au positionnement habituel des institutions de l'UE en faveur de la modernisation de l'agriculture et du marché unique est notable et bien accueillie. Les réformes successives de la PAC ouvrent une fenêtre d'opportunité pour la mise à l'agenda des CACP dans les PDR.

Cet avis, retenu par le règlement UE $n^{\circ} 1305 / 2013$ relatif au soutien au développement rural par le Feader, affirme que les CACP améliorent la compétitivité des producteurs primaires du fait de leur intégration dans la chaîne agroalimentaire. Les mesures portant sur les conseils peuvent désormais porter sur des questions spécifiques telles le développement des CACP. Dans les aides aux investissements physiques, l'efficacité du secteur de la commercialisation et de la transformation des produits agricoles est visée. La mise en place de petites installations de transformation et de commercialisation dans le cadre des CACP est prévue. Enfin, si au cours de la période de programmation 2007-2013, le seul type de coopération clairement soutenu dans les PDR était la mise au point de nouveaux produits, un soutien à la coopération horizontale et verticale entre les acteurs de la chaîne d'approvisionnement, ainsi qu'aux actions de promotion dans un contexte local, montre une réelle ouverture aux CACP (Parlement européen et Conseil, 2013). 
15 Afin de contribuer à la réalisation des priorités de l'UE en matière de développement rural, les États membres peuvent inclure dans leurs PDR des sous-programmes thématiques qui répondent à des besoins spécifiques. Ils peuvent concerner les jeunes agriculteurs, les petites exploitations, les zones de montagne, les femmes dans l'espace rural, l'atténuation des changements climatiques et aussi les CACP ${ }^{5}$. La liste indicative des mesures et opérations d'intérêt associées aux CACP intègre : la coopération, la mise en place de groupements de producteurs, le dispositif Leader, les systèmes de qualité applicables aux produits agricoles et aux denrées alimentaires, les services de base et rénovation des villages dans les zones rurales, les investissements physiques, le transfert de connaissances et actions d'information, ainsi que les services de conseil, d'aide à la gestion agricole et de remplacement sur l'exploitation (Parlement européen et Conseil, 2013).

\section{Un cadre d'analyse théorique et opérationnel}

Le fort développement des CACP, le cadre posé par la PAC 2014-2020 et la loi de modernisation de l'action publique territoriale et d'affirmation des métropoles de 2014 - qui confie aux conseils régionaux la gestion de la majorité des fonds européens en France - rendent d'actualité le débat sur les finalités et les moyens des politiques publiques adoptées. La question du changement a toujours été présente dans l'analyse des politiques publiques. Comme indiqué par Muller (2005), d'une certaine façon, on peut dire que les interrogations sur le changement sont à l'origine même de l'analyse des politiques publiques, qui se définissent toujours par rapport au changement que l'on cherche à freiner ou bien à promouvoir en lien avec un problème à résoudre et une population cible.

\subsection{Une grille de compréhension du fonctionnement des dispositifs d'intervention publique}

17 Reste que l'examen des politiques publiques peut s'appréhender sous différents angles. Knoepfel et al. (2015) distinguent trois courants de pensée : (1) l'analyse centrée sur les théories de l'État (2) l'évaluation des résultats et des effets, et (3) le courant qui cherche à expliquer le fonctionnement de l'action politique. C'est à ce dernier que nous nous attachons dans cet article. L'analyse de politiques publiques constitue alors non pas le révélateur d'un fonctionnement général du système politique et de mesure de l'efficience des mesures prises, mais un moyen de comprendre les modes opératoires et la logique d'action.

18 Nous adoptons la définition de politique publique proposée par ces auteurs, soit «un enchaînement de décisions ou d'activités, intentionnellement cohérentes, prises par des acteurs, publics et parfois privés, dont les ressources, les attaches institutionnelles et les intérêts varient, en vue de résoudre de manière ciblée un problème défini politiquement comme collectif. Cet ensemble de décisions et d'activités donne lieu à des actes formalisés, de nature plus ou moins contraignante, visant à modifier le comportement de groupes sociaux supposés à l'origine du problème collectif à résoudre, dans l'intérêt de groupes sociaux qui subissent les effets négatifs du problème en question " (Knoepfel et al., $2015: 42$ ).

19 Comme indiqué par Guérin (2006), nous partons du principe que la compréhension des mécanismes de soutien est un élément indispensable pour mettre en exergue les enjeux 
auxquels sont confrontés les acteurs, les processus de développement, les leviers d'action publique et les limites des interventions possibles ou envisagées. La politique publique suit ainsi une représentation séquentielle, soit un flux continu de décisions et de procédures (Muller, 1990 ; Knoepfel et al., 2015). Un cycle allant de l'émergence d'un problème, la mise en agenda, la formulation et adoption du programme, la mise en œuvre et l'évaluation ressort de la littérature.

Nous avons démarré notre réflexion par l'appréhension de la mise en agenda des CACP dans le cadre des politiques publiques française et européenne de développement rural. Nous allons ensuite centrer notre étude empirique sur les programmes adoptés par les PDR Occitanie favorables au développement des CACP.

Pour répondre aux objectifs UE retenus, un programme d'action prévisionnel qui permet de l'atteindre, formé d'une ou plusieurs actions, est structuré dans le temps. Des ressources de diverses natures (humaines, matérielles, financières, immatérielles) sont affectées à la réalisation du programme d'action. Enfin, un ensemble de règles décisionnelles déterminent non plus le contenu des actions à mener, mais la manière dont les décisions seront prises selon les éventualités rencontrées. De ce fait, nous regardons les actes formalisés des leviers actionnés, grâce aux appels à projets permanents ou restreints à une ou plusieurs périodes. Les appels à projets présentent les modalités d'intervention et de sélection des projets. Les grilles de notation, construites à partir des éléments décrits dans le PDR, permettent d'attribuer une note globale qui sert à la sélection des projets. Si les appels à projets ne permettent pas d'avoir une vision des projets sélectionnés et en développement, il est possible d'avoir un aperçu de l'importance accordée par les Régions aux actions liées aux CACP.

\subsection{Comprendre les logiques d'intervention des PDR Midi-Pyrénées et Languedoc-Roussillon}

Dans le contexte français d'organisation décentralisée pour la période 2014-2020, 27 programmes régionaux ont été créés. En effet, ils ont été constitués avant la Nouvelle organisation territoriale de la République $\left(\mathrm{NOTRe}^{6}\right)$ qui a redéfini les 13 régions métropolitaines, parmi lesquelles la Région Occitanie, qui regroupe désormais les PDR de deux anciennes régions : Midi-Pyrénées et Languedoc-Roussillon.

L'analyse comparée des mesures des PDR Midi-Pyrénées et Languedoc-Roussillon 2014-2020 permet de recenser la variété des initiatives publiques volontaristes adoptées en direction du développement des CACP. Un premier repérage des mesures, sousmesures et types d'opérations incluant les CACP a été fait grâce à la consultation des résultats d'une analyse thématique transversale des PDR des régions françaises pour la période 2014-2020, réalisée par la plateforme statistique de l'observatoire du développement rural (ODR)7 . Une recherche complémentaire a été faite sur les corpus originaux des PDR Midi-Pyrénées et Languedoc-Roussillon à l'aide de différents mots clés : vente directe, vente à la ferme, circuit court, circuit de proximité, plateforme d'achat, point de vente collectif, circuit d'approvisionnement, etc.

Une étude plus approfondie des types d'opérations pré-identifiées a permis de saisir dans la programmation des PDR les aspects impliquant les CACP. Comme le proposent Pivot et Mondot (2005), trois variables centrales ont été retenues : l'objet de l'opération, les bénéficiaires et les modes d'intervention (investissement, fonctionnement, etc.). C'est donc bien à un examen des dispositifs d'intervention, et non à une évaluation de 
leurs effets que nous nous attelons ici. Une attention particulière a été donnée au positionnement des références aux mots clés liés aux CACP. Pour appréhender les actes de mise en œuvre des PDR tournés vers le développement des CACP, nous avons étudié les appels à projets 2015 et 2016. On distingue un appel à projets par type d'opération. Ils regroupent les conditions d'application de la mesure tels les objectifs, les modalités de sélection (calendrier, critères de recevabilité, engagements des bénéficiaires, conditions d'éligibilité, sélection des projets, et grille de notation), et enfin les modalités de mise en œuvre (programmation et sanctions applicables). Un intérêt particulier est donné à la grille de notation, car elle permet d'identifier les thèmes concernant les CACP et leur importance dans la sélection des projets. Une grille de lecture a été ainsi établie en suivant six variables: la mesure, le type d'opération, le nombre et les thèmes relatifs aux CACP, les points/notations attribués, soit le minimal et le maximum. Pour situer l'ampleur des notes par thème, la somme minimale à obtenir pour déposer l'appel à projets a été identifiée.

\section{Les PDR d'Occitanie : une comparaison des mesures portant sur les CACP en Midi-Pyrénées et Languedoc-Roussillon}

L'élaboration des PDR s'appuie sur un diagnostic des besoins à couvrir sur le territoire du programme. L'objectif est d'établir parmi les priorités et mesures européennes, celles les plus adéquates et cohérentes avec les objectifs de la Région. Comme démontré dans le tableau 1, sur les 20 mesures présentes dans la politique rurale européenne, la France s'engage à en développer 17. Aucun des PDR n'a retenu la mesure 9, « mise en place de groupements de producteurs ", qui intègre pourtant des soutiens aux CACP.

Tableau 1. Mesures retenues par la France et les PDR Midi-Pyrénées et Languedoc-Roussillon (2014-2020)

\begin{tabular}{|c|c|c|c|c|c|c|c|c|c|}
\hline \multirow{2}{*}{$\begin{array}{l}\text { Mesures du Règlement du Développement Rural } 3 \\
\text { Intitulé }\end{array}$} & \multicolumn{6}{|c|}{ Priorité du RDR 3} & \multirow[t]{2}{*}{ France } & \multirow[t]{2}{*}{ MP } & \multirow[t]{2}{*}{ LR } \\
\hline & P1 & P2 & P3 & P4 & P5 & P6 & & & \\
\hline $\begin{array}{l}\text { Mesure 1: Transfert de connaissances et actions } \\
\text { d'information }\end{array}$ & $\mathrm{X}$ & & & & & & & & \\
\hline $\begin{array}{l}\text { Mesure } 2 \text { : Service de conseil, service d'aide à la gestion } \\
\text { agricole et service de remplacement sur l'exploitation }\end{array}$ & $\mathrm{x}$ & $\mathrm{x}$ & $\mathrm{X}$ & $\mathrm{X}$ & $\mathrm{X}$ & $\mathrm{X}$ & & & \\
\hline $\begin{array}{l}\text { Mesure } 3 \text { : Système de qualité applicables aux produits } \\
\text { agricoles et aux denrées alimentaires }\end{array}$ & & $\mathrm{x}$ & & & & & & & \\
\hline Mesure 4 : Investissements physiques & $\mathrm{x}$ & $\mathrm{x}$ & $\mathrm{X}$ & $\mathrm{X}$ & $\mathrm{X}$ & $\mathrm{X}$ & & & \\
\hline $\begin{array}{l}\text { Mesure } 5: \text { Reconstitution du potentiel de production } \\
\text { agricole endommagé par des catastrophes naturelles et } \\
\text { mesure de prévention }\end{array}$ & & & $\mathrm{X}$ & & & & & & \\
\hline $\begin{array}{l}\text { Mesure 6: Développement des exploitations et des } \\
\text { entreprises }\end{array}$ & $x$ & $\mathrm{X}$ & $\mathrm{X}$ & $\mathrm{X}$ & $\mathrm{X}$ & $\mathrm{x}$ & & & \\
\hline $\begin{array}{l}\text { Mesure } 7: \text { Services de base de rénovation des villages } \\
\text { dans les zones rurales }\end{array}$ & & & & & & $\mathrm{X}$ & & & \\
\hline
\end{tabular}




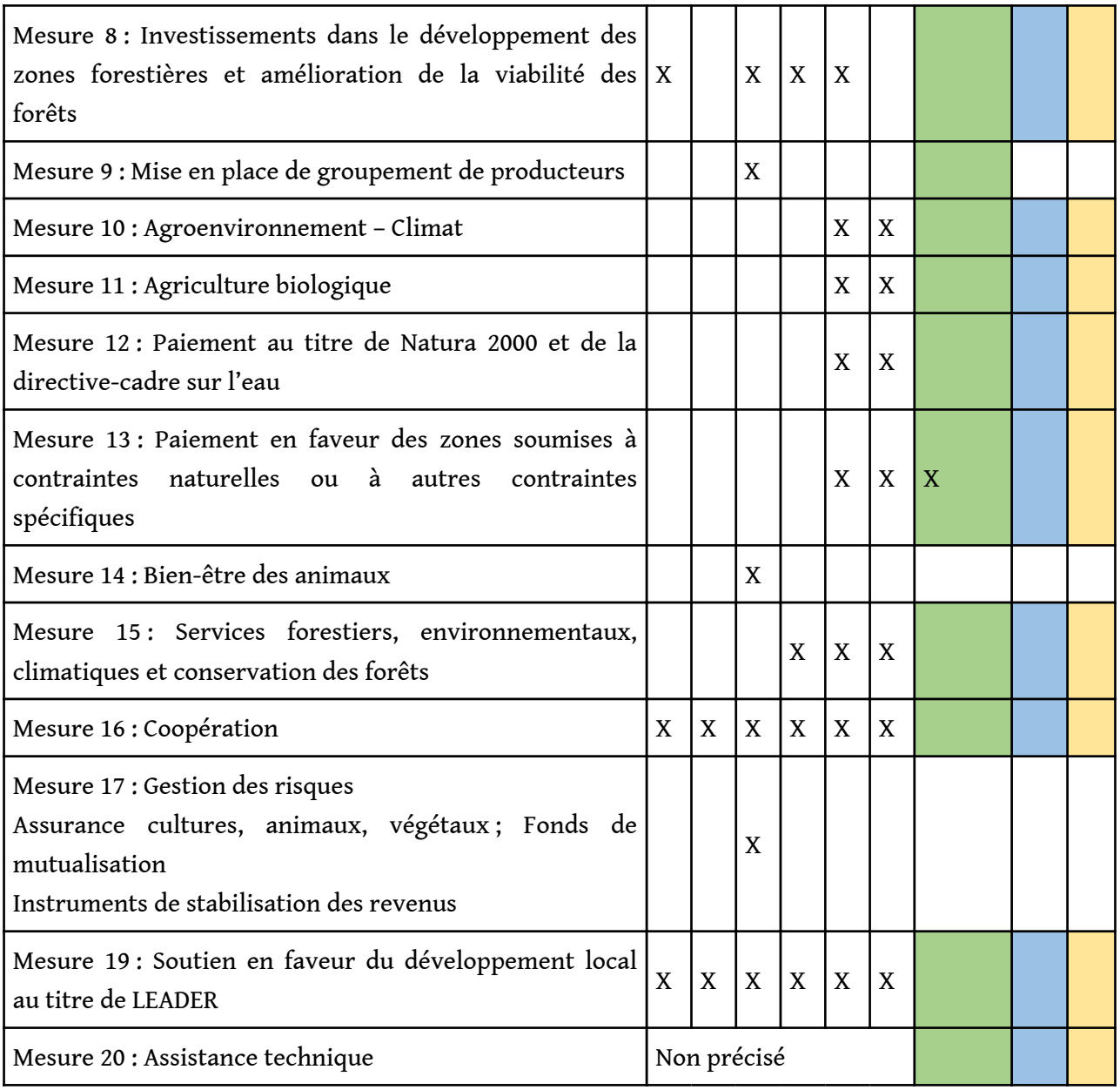

La mesure 18 est spécialement dédiée aux paiements directs nationaux complémentaires pour la Croatie.

Source : Les auteurs, adapté du Réseau rural français (2017)

Les budgets Feader des régions Languedoc-Roussillon et Midi-Pyrénées soulignent un écart substantiel : 597 millions contre 1,307 milliard d'euros. Midi-Pyrénées bénéfice de la plus grande enveloppe nationale pour la programmation 2014-2020. Les deux anciennes régions ont retenu les mêmes mesures, à l'exception de celle portant sur les services de conseil, d'aide à la gestion agricole et de remplacement sur l'exploitation, non retenue par Languedoc-Roussillon.

\subsection{Les mesures intégrant un soutien aux CACP en Languedoc- Roussillon et Midi-Pyrénées}

Trois mesures inscrivent des soutiens aux CACP dans les PDR d'Occitanie. Elles portent sur les investissements physiques; les aides à l'installation et au développement des exploitations et des entreprises; et la coopération territoriale (cf. figure 2). Grâce à l'analyse textuelle complémentaire portée sur les corpus originaux, il a été possible d'identifier les projets éligibles, les modalités d'aide et les critères de sélection.

La mesure 4 vient en soutien aux investissements des exploitations agricoles, afin de les accompagner vers un renforcement de leur compétitivité et des pratiques répondant à l'agroécologie. Quatre types d'opérations font référence aux CACP. Deux sont destinées 
aux soutiens aux exploitations engagées dans une démarche de valorisation et/ou à la transformation et à la commercialisation des produits de la ferme. Dans le premier, les exploitations agricoles et les groupements de producteurs peuvent recourir à des subventions d'investissement; dans le second à des investissements matériels et de fonctionnement. Les soutiens peuvent être destinés à la mise en place de ventes directes et à la ferme, ainsi qu'à la structuration des circuits de commercialisation collectifs. En Languedoc-Roussillon, un des critères de sélection demande l'adhésion à une démarche collective de circuit court reconnue par la Région.

Tableau 2. Prise en compte des CACP dans les opérations des PDR d'Occitanie

\begin{tabular}{|c|c|c|c|}
\hline Mesure & Objet & $\begin{array}{l}\text { Type } \\
\text { d'opération }\end{array}$ & $\begin{array}{l}\text { Positionnement des CACP dans le } \\
\text { PDR }\end{array}$ \\
\hline \multirow{6}{*}{$\begin{array}{l}\text { 4: Investissements } \\
\text { physiques }\end{array}$} & \multirow{2}{*}{$\begin{array}{l}\text { Investissements } \\
\text { spécifiques des } \\
\text { exploitations engagées } \\
\text { dans une démarche de } \\
\text { valorisation }\end{array}$} & MP 4.1.2 & $\begin{array}{l}\text { Critères de sélection : } \\
\text { "développement de circuits courts et } \\
\text { de proximité, commercialisation en } \\
\text { vente directe " }\end{array}$ \\
\hline & & LR 4.1.1 & $\begin{array}{l}\text { Critères de sélection: "Adhésion à } \\
\text { une démarche collective circuits courts } \\
\text { reconnue par la Région" }\end{array}$ \\
\hline & \multirow{2}{*}{$\begin{array}{l}\text { Investissements des } \\
\text { exploitations liés à } \\
\text { la transformation et à } \\
\text { la commercialisation }\end{array}$} & MP 4.2.1 & $\begin{array}{l}\text { Objectif: «mise en place de circuits } \\
\text { de proximité favorisant le } \\
\text { rapprochement producteurs/ } \\
\text { consommateurs" } \\
\text { Mode d'intervention: «soutien à } \\
\text { l'investissement afin de favoriser la } \\
\text { transformation et la } \\
\text { commercialisation en circuit court» }\end{array}$ \\
\hline & & LR 4.2.1 & $\begin{array}{l}\text { Mode d'intervention: "soutien } \\
\text { pour construction point de vente à la } \\
\text { ferme, ou matériels/équipements } \\
\text { pour vente en circuit court " } \\
\text { Critères de sélection: "adhésion à } \\
\text { une démarche collective circuits } \\
\text { courts reconnue par la Région» }\end{array}$ \\
\hline & \multirow[t]{2}{*}{$\begin{array}{l}\text { Investissements dans les } \\
\text { Cuma }\end{array}$} & MP : 4.1.6 & $\begin{array}{l}\text { Thématique : "organisation de } \\
\text { l'offre dans le cadre de circuits courts } \\
\text { de distribution " Critère de sélection: } \\
\text { "transformation des produits pour } \\
\text { le développement des circuits } \\
\text { courts de valorisation" }\end{array}$ \\
\hline & & LR : 4.1.2 & $\begin{array}{l}\text { Critères de sélection: "Cuma de } \\
\text { transformation avec destination des } \\
\text { produits en circuit de proximité » }\end{array}$ \\
\hline
\end{tabular}




\begin{tabular}{|c|c|c|c|}
\hline & $\begin{array}{l}\text { Investissements dans les } \\
\text { entreprises de } \\
\text { transformation et de } \\
\text { commercialisation des } \\
\text { produits agricoles }\end{array}$ & LR : 4.2.2 & $\begin{array}{l}\text { Objectif : « aptitude à conquérir de } \\
\text { nouveaux marchés, en particulier à } \\
\text { l'export ou en circuit court» }\end{array}$ \\
\hline \multirow{2}{*}{$\begin{array}{l}6: \quad \text { Aides à } \\
\text { l'installation et au } \\
\text { développement des } \\
\text { exploitations et des } \\
\text { entreprises }\end{array}$} & $\begin{array}{l}\text { Dotation aux jeunes } \\
\text { agriculteurs }\end{array}$ & MP : 6.1.1 & $\begin{array}{l}\text { Majoration: "projet générateur de } \\
\text { valeur ajoutée visant une meilleure } \\
\text { valorisation des produits (produits } \\
\text { SIQO, commercialisation en circuits } \\
\text { courts)» }\end{array}$ \\
\hline & $\begin{array}{l}\text { Diversification des } \\
\text { exploitations agricoles } \\
\text { vers des activités non } \\
\text { agricoles }\end{array}$ & LR : 6.4 .1 & $\begin{array}{l}\text { Critères de sélection: "projet } \\
\text { s'inscrivant dans une stratégie } \\
\text { collective de filière ou de } \\
\text { territoire "; "adhésion à une } \\
\text { démarche collectivecircuits courts, } \\
\text { participation à une formation } \\
\text { action territoriale sur } \\
\text { l'agritourisme ou les circuits courts " }\end{array}$ \\
\hline 16 : Coopération & Coopération & LR : 16.7.1 & $\begin{array}{l}\text { Thématique: «structuration et } \\
\text { développement des filières } \\
\text { économiques du territoire: filière } \\
\text { bois, circuits courts et de proximité, } \\
\text { agritourisme et approvisionnement } \\
\text { des structures collectives" }\end{array}$ \\
\hline
\end{tabular}

Les deux autres types d'opérations sont liés aux Cuma ou aux entreprises de transformation et commercialisation des produits agricoles. Tandis que la première vise dans les deux PDR à favoriser la transformation des produits pour le développement des CACP, la seconde implique les entreprises en Languedoc-Roussillon dans l'approvisionnement des produits agricoles régionaux et locaux. Les soutiens à l'approvisionnement local peuvent intégrer les critères d'éco-conditionnalité.

La mesure 6 vise globalement à favoriser l'installation des jeunes agriculteurs (JA). Deux types d'opérations intègrent des aspects de commercialisation en CACP : (1) en Midi-Pyrénées, les dotations visent à valoriser leurs productions; (2) en LanguedocRoussillon, elles favorisent la diversification en direction d'activités non agricoles. La première offre aux agriculteurs des subventions d'investissements matériels. Les CACP sont pris en compte dans le cadre des modulations, au même titre que les produits sous signe officiel de la qualité et de l'origine (SIQO). Le deuxième offre des subventions d'investissement et de fonctionnement à l'agrotourisme, la transformation ou la commercialisation. À nouveau en Languedoc-Roussillon, les critères de sélection intègrent l'adhésion à des CACP reconnus par la Région et ajoutent la participation à une formation sur l'agritourisme ou les CACP.

31 La coopération territoriale est visée dans le cadre de la mesure 16. Une des thématiques concerne la structuration et le développement des filières économiques en LanguedocRoussillon. Elle ouvre son champ d'action aux CACP et à l'approvisionnement des structures collectives. Les bénéficiaires sont divers et comprennent les collectivités, les coopératives, les organismes agricoles, les structures des Parcs naturels régionaux, etc. 
Les dépenses de mise en place des coopérations sont financées, ainsi que la communication et les animations opérationnelles et relatives à l'émergence des stratégies locales de développement. L'appui au développement des CACP intègre une logique multipartenariale.

\subsection{Un soutien timide aux CACP, mais plus marqué dans les appels à projets de Languedoc-Roussillon}

Comme précédemment indiqué, les PDR Midi-Pyrénées et Languedoc-Roussillon regroupent l'ensemble des intentions politiques élaborées en fonction des domaines prioritaires européens, des mesures actées en France et des besoins de chaque exRégion. On y retrouve un ensemble d'éléments qui décrivent les intentions politiques et le cadre pour le passage à l'acte. Ils sont établis par les Régions une fois que les PDR sont adoptés. Les appels à projets 2014-2020 sont formés par de nombreux thèmes (entre 4 et 13), dont une petite partie implique explicitement les CACP. Pour autant, aucune mesure n'est restreinte aux seuls CACP. Au regard des grilles de notation, nous avons identifié trois classes d'appels à projets selon la contribution des actions en lien avec les CACP dans l'obtention du nombre minimum des points nécessaires pour le dépôt du dossier: classe 1 - susceptible de valider le dépôt du dossier; classe 2contribue de manière importante ; et classe 3 - l'importance est mineure.

La mesure 4 portant sur les investissements physiques rassemble des thématiques permettant de mettre les CACP au cœur des projets. Comme démontré dans le Tableau 3, les projets d'investissements dans les Cuma de transformation destinées à une valorisation en CACP permettent dans les deux PDR d'obtenir le minimum des points requis à la validation du dépôt du dossier. En Midi-Pyrénées, deux autres thématiques sur onze, touchant cette fois les investissements des exploitations pour la mise en œuvre des projets de transformation et de commercialisation, se retrouvent dans le même cas de figure. Si ces ponctuations sont encourageantes pour le développement des $\mathrm{CACP}$, il faut prendre en compte le fait que d'autres éléments peuvent atteindre jusqu'à 1000 points.

Tableau 3. Classe 1 - les investissements physiques en lien avec les CACP en mesure de valider un appel à projets

\begin{tabular}{|c|c|c|c|c|c|c|}
\hline Mesure & Objet & $\begin{array}{l}\text { Type } \\
\text { d'opération }\end{array}$ & $\begin{array}{l}\text { Nombre } \\
\text { total de } \\
\text { thèmes }\end{array}$ & $\begin{array}{l}\text { Thème(s) portant } \\
\text { sur les CACP }\end{array}$ & $\begin{array}{l}\text { Points } \\
\text { attribués }\end{array}$ & $\begin{array}{l}\text { Minimale } \\
\text { pour la } \\
\text { validation }\end{array}$ \\
\hline $\begin{array}{l}\text { Investissements } \\
\text { physiques }\end{array}$ & 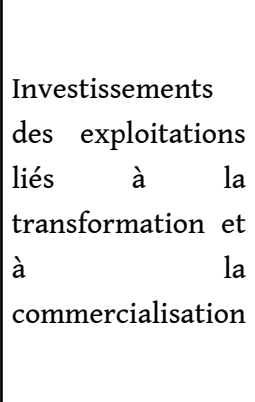 & MP 4.2.1 & 11 & $\begin{array}{l}\text { T.6: } \\
\text { «Projet concernant } \\
\text { la transformation et } \\
\text { la } \\
\text { commercialisation " } \\
\text { T.8: } \\
\text { «Projet } \\
\text { uniquement de } \\
\text { commercialisation » }\end{array}$ & $\begin{array}{ll}\text { T.6: } & 50 \\
\text { pts } & \\
\text { T.8: } & 40 \\
\text { pts } & \end{array}$ & 40 \\
\hline
\end{tabular}




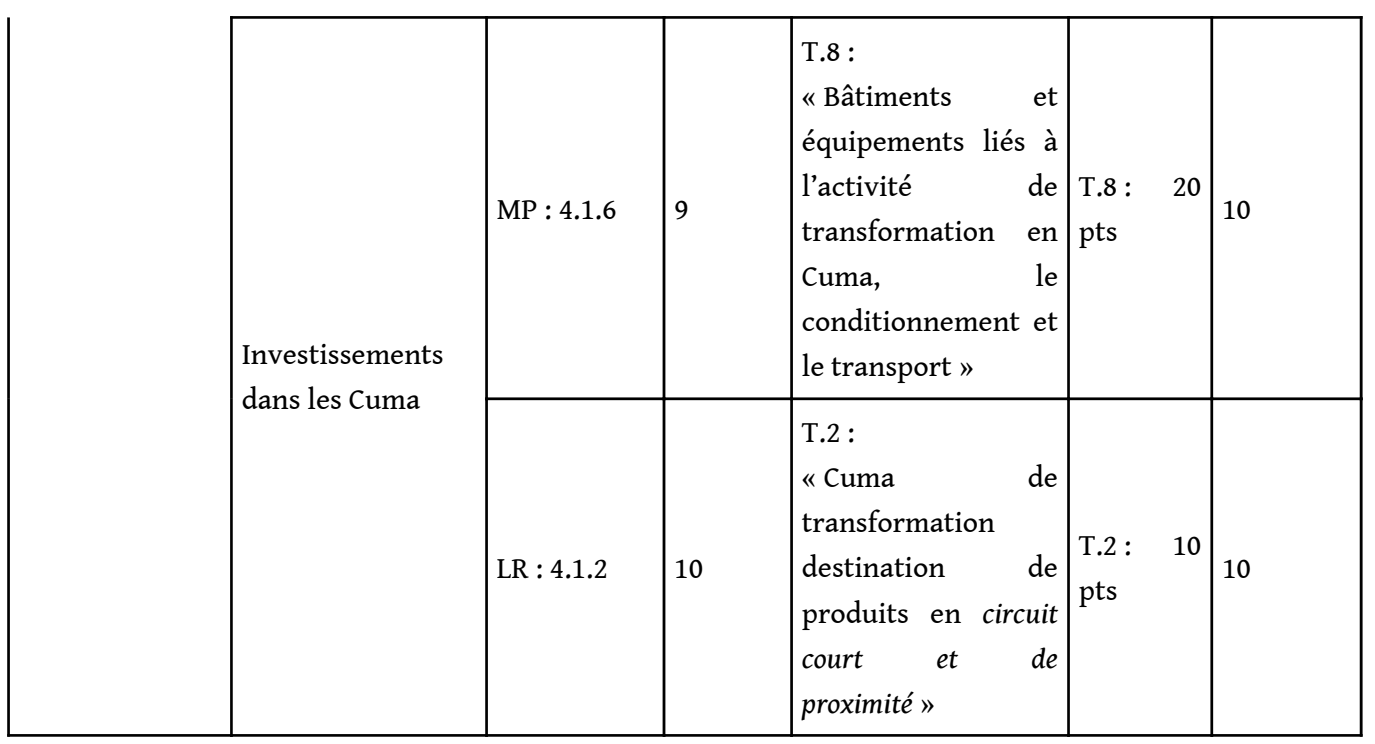

En Languedoc-Roussillon, certains investissements physiques ne comptabilisent pas le minimum de points pour la validation de l'appel à projets, mais peuvent contribuer de manière considérable à l'obtention du minimum de points nécessaires pour le dépôt du projet (cf. tableau 4). Les projets axés sur l'alimentation de proximité et de qualité par l'utilisation des ressources productives du territoire confèrent 50 points. Le même montant est obtenu pour les filières laitières, viande et apiculture qui développent un projet avec une dimension territoriale et/ou collective portant sur la valorisation locale de la production, le développement d'une stratégie commerciale territorialisée ou basée sur les CACP. Les boutiques collectives de producteurs pèsent fortement dans la grille de notation car 70 points leur sont associés, presque le minimum nécessaire pour valider l'appel à projets (80 points).

Tableau 4. Classe 2 - les investissements physiques en lien avec les CACP ayant une contribution modérée à la validation d'un appel à projets

\begin{tabular}{|l|l|l|l|l|l|l|}
\hline Mesure & Objet & $\begin{array}{l}\text { Type } \\
\text { d'opération }\end{array}$ & $\begin{array}{l}\text { Nombre } \\
\text { total de } \\
\text { thèmes }\end{array}$ & $\begin{array}{l}\text { Thème portant sur } \\
\text { les CACP }\end{array}$ & $\begin{array}{l}\text { Points } \\
\text { attribués }\end{array}$ & $\begin{array}{l}\text { Minimale } \\
\text { pour la } \\
\text { validation }\end{array}$ \\
\hline
\end{tabular}




\begin{tabular}{|c|c|c|c|c|c|c|}
\hline \multirow[b]{2}{*}{$\begin{array}{l}\text { Investissements } \\
\text { physiques }\end{array}$} & 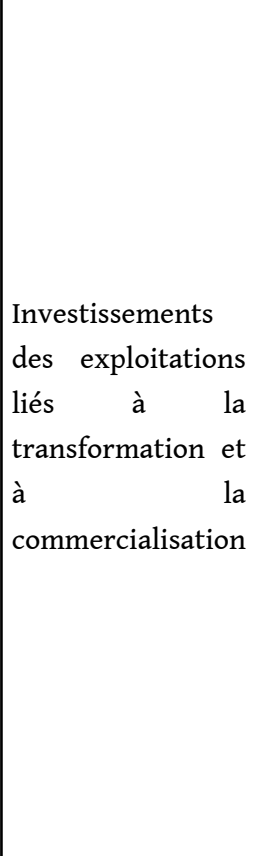 & LR 4.2.1 & 9 & 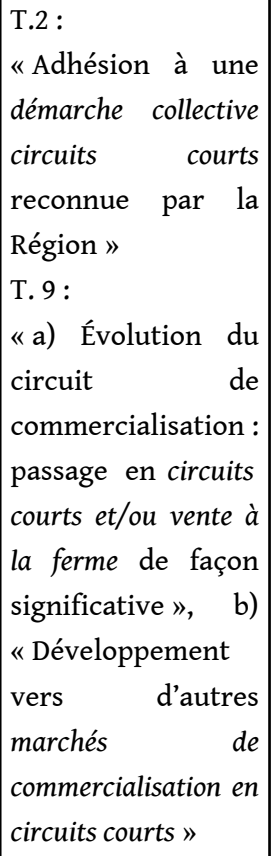 & $\begin{array}{l}\text { T. } 2: 10 \text { pts } \\
\text { T. } 9: \\
\text { a) } 15 \text { pts, } \\
\text { b) } 5 \text { pts } \\
\text { a et b non } \\
\text { cumulables }\end{array}$ & 30 \\
\hline & $\begin{array}{l}\text { Investissements } \\
\text { dans les } \\
\text { entreprises de } \\
\text { transformation et } \\
\text { de } \\
\text { commercialisation } \\
\text { des produits } \\
\text { agricoles }\end{array}$ & LR : 4.2.2 & 13 & $\begin{array}{l}\text { T.4: «Projet axé } \\
\text { sur l'alimentation de } \\
\text { proximité et de } \\
\text { qualité (développer } \\
\text { l'utilisation des } \\
\text { ressources de } \\
\text { productives du } \\
\text { territoire, viser des } \\
\text { marchés } \\
\text { proximité... " } \\
\text { T.5: } \\
\text { développant une } \\
\text { dimension } \\
\text { territoriale et/ou } \\
\text { collective: } \\
\text { Valorisation locale } \\
\text { de la production, } \\
\text { développement } \\
\text { d'une stratégie } \\
\text { commerciale } \\
\text { territorialisée ou } \\
\text { basée sur des } \\
\text { circuits courts ou de } \\
\text { proximité » } \\
\text { T.6: } \\
\text { «Projet portant } \\
\text { sur la création ou } \\
\text { le développement } \\
\text { d'une boutique } \\
\text { collective } \\
\text { producteurs » }\end{array}$ & $\begin{array}{l}\text { T.4: } 50 \text { pts } \\
\text { T.5: } 50 \text { pts } \\
\text { T.6: } 70 \text { pts }\end{array}$ & 80 \\
\hline
\end{tabular}



coopération territoriale. Les thématiques portant sur les CACP ont une contribution mineure à la validation de l'appel à projets (cf. tableau 5). Dans les deux anciennes Régions, les dotations aux JA prévoient des modulations limitées à 30 points sur les 300 nécessaires pour le dépôt d'un dossier. En Languedoc-Roussillon, les CACP peuvent s'inscrire dans la promotion de la diversification des exploitations vers des activités non agricoles. L'adhésion à une démarche collective circuits courts reconnue par la Région ou la participation à une formation sur les CACP rassemblent chacune 10 points sur 80. Enfin, les CACP font partie des éléments en lien avec la coopération territoriale (mesure 16) en Languedoc-Roussillon. La structuration et le développement des CACP et l'approvisionnement des structures collectives figurent parmi les filières économiques du territoire ( 5 des 80 points minimums requis).

Tableau 5. Classe 3 - les thématiques en lien avec les CACP ayant une contribution réduite à la validation du dépôt d'un dossier PDR

\begin{tabular}{|c|c|c|c|c|c|c|}
\hline Mesure & Objet & $\begin{array}{l}\text { Type } \\
\text { d'opération }\end{array}$ & $\begin{array}{l}\text { Nombre } \\
\text { total de } \\
\text { thèmes }\end{array}$ & $\begin{array}{l}\text { Thème portant sur les } \\
\text { CACP }\end{array}$ & $\begin{array}{l}\text { Points } \\
\text { attribués }\end{array}$ & $\begin{array}{l}\text { Minimale } \\
\text { pour la } \\
\text { validation }\end{array}$ \\
\hline & Dotation aux & MP : 6.1.1 & 4 & $\begin{array}{l}\text { T.4 : } \\
\text { «Nombre } \\
\text { modulations } \\
\text { sollicitées : projet } \\
\text { générateur de valeur } \\
\text { ajoutée visant une } \\
\text { meilleure } \\
\text { valorisation des } \\
\text { produits (produits } \\
\text { SIQO, } \\
\text { commercialisation en } \\
\text { circuits courts)" }\end{array}$ & $\begin{array}{lr}\text { T.4 : } \\
10 \quad \mathrm{pts} / \\
\text { modulation } \\
\text { dans } & \text { la } \\
\text { limite } & \text { de } \\
30 \mathrm{pts} & \end{array}$ & 300 \\
\hline $\begin{array}{l}6: \\
\text { Aides à } \\
\text { l'installation et } \\
\text { au } \\
\text { développement } \\
\text { des } \\
\text { exploitations } \\
\text { et entreprises des } \\
\text { entri }\end{array}$ & agriculteurs & LR : 6.1 .1 & 4 & $\begin{array}{l}\text { T.4 : } \\
\text { «Nombre } \\
\text { modulations } \\
\text { sollicitées : projet } \\
\text { générateur de valeur } \\
\text { ajoutée visant une } \\
\text { meilleure } \\
\text { valorisation des } \\
\text { produits (produits } \\
\text { SIQO, } \\
\text { commercialisation en } \\
\text { circuits courts)" }\end{array}$ & $\begin{array}{ll}\text { T.4: } \\
10 \text { pts } \\
\text { modulation } \\
\text { dans } \quad \text { la } \\
\text { limite de } \\
30 \text { pts }\end{array}$ & 300 \\
\hline
\end{tabular}




\begin{tabular}{|c|c|c|c|c|c|c|}
\hline & $\begin{array}{l}\text { Diversification } \\
\text { des } \\
\text { exploitations } \\
\text { agricoles vers } \\
\text { des activités } \\
\text { non agricoles }\end{array}$ & LR : 6.4 .1 & 10 & 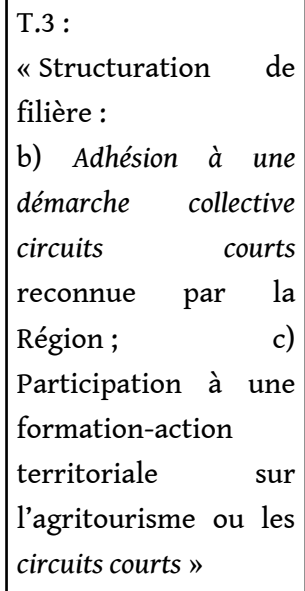 & $\begin{array}{l}\text { T.3 : } \\
\text { b) } 10 \mathrm{pts}, \mathrm{c}) \\
10 \mathrm{pts}\end{array}$ & 80 \\
\hline $\begin{array}{l}16: \\
\text { Coopération } \\
\text { territoriale }\end{array}$ & $\begin{array}{l}\text { Coopération } \\
\text { territoriale }\end{array}$ & LR : 16.7.1 & 6 & $\begin{array}{l}\text { T.2: } \\
\text { «Structuration et } \\
\text { développement des } \\
\text { filières économiques } \\
\text { du territoire: filière } \\
\text { bois, circuits courts et } \\
\text { de proximité, } \\
\text { agritourisme, et } \\
\text { approvisionnement } \\
\text { des structures } \\
\text { collectives » }\end{array}$ & $\begin{array}{l}\text { T.2: } \\
5 \text { pts }\end{array}$ & 80 \\
\hline
\end{tabular}

Globalement, les CACP peuvent jouer un rôle important ou modéré dans les projets d'investissements physiques. Le PDR Languedoc-Roussillon présente un appui plus marqué à certaines filières, à la création d'un circuit court spécifique, en l'occurrence les boutiques collectives de producteurs, ainsi qu'aux organisations déjà reconnues dans la région. Les CACP ont une contribution réduite dans les grilles de notation des mesures de coopération territoriale, et d'aide à l'installation et au développement des exploitations et des entreprises.

\section{Les perspectives pour la PAC et les PDR post-2020}

37 L'étude des PDR Midi-Pyrénées et Languedoc-Roussillon montre que le transfert de compétence de l'État vers les Régions se traduit, en matière de soutien aux CACP, par des interventions limitées à trois mesures: les investissements physiques, le développement des exploitations agricoles et des entreprises, ainsi que la coopération. Trois autres mesures portant au niveau européen sur les CACP n'ont pas été mobilisées dans les PDR de la Région Occitanie. Trois autres n'indiquent pas explicitement les CACP dans les objectifs 2014-2020, mais pourraient trouver des convergences.

\subsection{Des mesures à reconduire}

Tout d'abord, parmi les mesures à reconduire figure la mesure 1 qui porte sur le transfert de connaissance et les actions d'information, soit le renforcement du potentiel humain et des compétences des acteurs. Des échanges sur la participation aux 
CACP à travers des séjours d'agriculteurs dans d'autres fermes, à l'échelle de l'UE, sont déjà prévus dans les mesures Feader. Le maintien de ce type d'action et sa mobilisation dans la prochaine programmation pourraient permettre aux agriculteurs de rechercher de nouvelles perspectives dans les actions entreprises par les pays voisins. l'exploitation (mesure 2) n'ont pas été retenus par les PDR Languedoc-Roussillon et n'impliquent pas des opérations portant sur les CACP en Midi-Pyrénées. Cependant, les conseils peuvent porter sur le développement des circuits courts. La mise en place d'activités de transformation et de commercialisation dans les exploitations pour augmenter la valeur ajoutée des produits agricoles est couverte par les multiples thématiques. Le conseil collectif peut être éligible sous certaines conditions et devrait être davantage impulsé dans la prochaine PAC.

La mesure 20 soutient la mise en réseaux des acteurs du développement rural, soit les représentants d'agriculteurs, les organismes de développement agricoles, les communes rurales, les associations, etc. Elle a un rôle important à jouer dans le soutien à l'échange d'expériences, la diffusion des bonnes pratiques et la communication auprès du grand public.

\subsection{Des mesures à explorer}

41 La mesure 3 porte sur le développement des produits agricoles issus de systèmes qui garantissent la qualité des produits via des signes officiels d'identification de la qualité, de l'origine, ainsi que toutes démarches de certification volontaire de produits agricoles reconnus par les autorités. Certains travaux démontrent que la présence de signes de qualité est importante dans les CACP. Selon l'enquête de Bouroullec et al. (2016) réalisée en Midi-Pyrénées, $36 \%$ des productions commercialisées dans les CACP sont en agriculture biologique $(\mathrm{AB})$ ou d'autres certifications officielles comme les AOP/ AOC, IGP ou le Label Rouge. La loi EGalim précédemment évoquée vient renforcer le rapprochement potentiel et déjà observé entre $\mathrm{CACP}$ et produits SIQO, et notamment les produits $\mathrm{AB}$. Le clivage entre les SIQO et les $\mathrm{CACP}$ doit être travaillé. Pour répondre aux ambitions de la loi EGalim, cette mesure pourrait continuer à appuyer les agriculteurs lors de leur entrée dans les systèmes de qualité. L'objectif est de réduire les freins économiques de la participation au système de qualité, notamment au cours des premières années. La toute récente feuille de route de l'UE "De la ferme à la table ", dans le cadre du Pacte vert, va dans le même sens en soutenant le développement de l'agriculture biologique et la réduction des engrais notamment.

La mesure 9 sur la mise en place de groupements et d'organisations de producteurs est également passible d'élargir les incitations pour le développement des CACP. Le dispositif encourage les agriculteurs à s'organiser collectivement afin de faire face aux défis du marché et de renforcer leur pouvoir de négociation. Comme indiqué par King et al. (2010) et Berti et Mulligan (2016), un défi récurrent auquel sont confrontés les agriculteurs est le manque d'infrastructures et de services de distribution pour leur permettre de profiter davantage de la demande croissante de produits cultivés localement et régionalement. L'organisation des agriculteurs et les actions collectives sont des facteurs clés pour améliorer l'accès des agriculteurs aux marchés, surtout sur les marchés publics. 

CACP. Les Groupes d'action locale (GAL) sont déjà une réalité en France. Plus de 40 ont été structurés en Occitanie pour renforcer la capacité des acteurs locaux à élaborer et mettre en œuvre des opérations et des procédures de sélection transparente et non discriminatoire. Les soutiens qui portent sur la préparation, mise en œuvre d'opérations et d'activités de coopération pourraient inscrire de manière plus explicite des actions qui permettent l'émergence et le développement des CACP collectifs. Nos travaux et d'autres permettent de souligner les difficultés auxquelles sont confrontés les CACP en termes de financement des frais de fonctionnement collectifs, soit les coûts des infrastructures commerciales et logistiques, ainsi que de ressources humaines. Le développement des CACP à travers la mise en place des GAL semble un élément important pour le renforcement d'une stratégie locale de développement, partenariale et ascendant à reconduire et renforcer dans la PAC post-2020. Ces dispositifs pourraient venir consolider la dimension territoriale des initiatives en CACP également portée par les PAT.

\section{Conclusion}

L'étude des PDR Midi-Pyrénées et Languedoc-Roussillon, établis avant la fusion des Régions, confirme les soutiens publics aux CACP. Ils s'inscrivent dans les mesures portant sur les investissements physiques et l'aide à l'installation, dans les deux PDR d'Occitanie, ainsi que la coopération dans le PDR Languedoc-Roussillon. Les soutiens sont encore timides. Seules deux thématiques citent spécifiquement les CACP (CUMA et coopération). Les mentions aux CACP sont davantage intégrées aux critères de sélection et s'inscrivent aussi dans les objectifs, mode d'intervention et majorations des opérations. Soulignons également que les mesures 4 et 6 bénéficient d'un cofinancement de l'État, interrogeant la logique de choix des orientations régionales : les moyens dédiés aux CACP ne constituent-ils qu'une mobilisation des financements étatiques ou cette complémentarité des financements autorise-t-elle une plus large palette d'interventions de la part des Régions dans un contexte de contrainte budgétaire resserrée?

Les moindres différences observées dans les PDR étudiés sont encourageantes pour la prochaine programmation de la PAC dont les négociations européennes se poursuivent sur la base d'un plan stratégique national établi pour sept ans. Cependant, le passage à un plan stratégique national rouvre les questionnements sur la diversité des modèles agricoles, les disparités territoriales, et l'adaptation de formes de redistribution au sein des Régions. Le degré d'autonomie laissé aux Régions constituera un point essentiel du dispositif d'intervention à venir, notamment en ce qui concerne le pilotage des fonds mobilisables pour soutenir les CACP.

La baisse du budget annoncée aura des conséquences sur le Feader. De nombreux points vont faire débat et influenceront les aides. Malgré cela, le soutien aux CACP plébiscité par les consommateurs, via la restauration collective biologique et locale, ainsi qu'à la transformation artisanale des produits fermiers semble prioritaire, notamment après la crise de la Covid-19. Le soutien aux CACP pourrait entre autres être axé sur plusieurs des neuf objectifs UE : assurer un revenu équitable aux agriculteurs; accroître la compétitivité; rééquilibrer les pouvoirs dans la chaîne d'approvisionnement alimentaire; soutenir le renouvellement des générations; et dynamiser et soutenir le

Développement durable et territoires, Vol. 12, n¹ | Mai 2021 
développement économique des zones rurales. La mobilisation de plusieurs mesures et une meilleure prise en compte des actions en lien avec le développement et consolidation des CACP dans les systèmes de notation des appels à projets est possible.

Enfin, les CACP peuvent être au carrefour des multiples dispositifs alloués au développement rural et se développer de plus en plus vers des actions et objectifs collectifs. Cependant, les actions collectives ascendantes et multipartenariales s'avèrent des processus complexes et parfois fragiles, pas nécessairement en phase avec les pratiques des agriculteurs. Les mécanismes de gouvernance à mettre en place sont essentiels pour garantir une implication pérenne des acteurs mobilisés dans ces démarches, assurer des mécanismes de prise des décisions efficaces, permettre la gestion des risques et des conflits. En ce sens, les organismes consulaires, instituts techniques et l'Organisme national à vocation agricole et rurale (Onvar) peuvent infléchir leurs pratiques pour constituer des relais à la mise en place de politiques en faveur des CACP sur les territoires. Reste à la prochaine programmation de la PAC à se donner les moyens de leur intégration efficace dans les dispositifs qui seront élaborés.

\section{BIBLIOGRAPHIE}

Berti G., Mulligan C., 2016, « Competitiveness of Small Farms and Innovative Food Supply Chains: the role of food hubs in creating sustainable regional and local food systems ", Sustainability, vol. 8, p. 1-31.

Brand C., Bricas N., Conaré D., Daviron B., Debru J., Michel L., Soulard Ch., 2017, Construire des politiques alimentaires urbaines. Concepts et démarches, Versailles, Quæ, collection « Update Sciences et Technologies ", 978-2-7592-2617-7.

Chaffotte L., Chiffoleau Y., 2007, « Vente directe et circuits courts : évaluations, définitions et typologie ", Les cahiers de l'observatoire CROC, $\mathrm{n}^{\circ} 1$, p. 1-8.

Comité des régions, 2011, « Avis de prospective relatif aux systèmes agroalimentaires locaux ", Journal officiel de l'Union européenne, C 104/1, 88 session plénière des 27 et 28 janvier 2011.

Décret $n^{\circ}$ 2019-1344 du 12 décembre 2019 modifiant certaines dispositions du code de la commande publique relatives aux seuils et aux avances, JORF $\mathrm{n}^{\circ} 0289 \mathrm{du} 13$ décembre 2019 , texte $n^{\circ} 21$.

Guérin M., 2006, «Évolution des objectifs et des principes d'intervention des politiques de développement rural sur le long terme », Ingénieries Eau-Agriculture-Territoires, numéro spécial sur les politiques rurales, p. 27-38.

Hérault-Fournier C., Prigent-Simonin A.-H., 2012, « Typologie des consommateurs en fonction de la proximité perçue en vente directe : Exploration de trois formes de vente : AMAP, Points de vente collectifs et Marchés », https://umr-moisa.cirad.fr/content/download/5508/37899/ version/1/file/Herault-Fournier,+Pringent-Simonin+JMAM+2012.pdf, consulté le 13/12/2016. 
Jouen M., Lorenzi F., 2014, « La dimension territoriale et politique des circuits courts alimentaires : représentations et enjeux dans le débat européen sur la politique agricole commune ", Science Eaux \& Territoires, n 13, p. 12-19, https://doi.org/10.3917/set.013.0012.

King, R. P., S. Hand M., DiGiacomo G., Clancy K., Gomez M., Hardesty S., Lev L., McLaughlin E. W., 2010, « Comparing the Structure, Size, and Performance of Local and Mainstream Food Supply Chains », Economic Research Report, United States Department of Agriculture, Econ. Res. Serv. June.

Kneafsey M., Venn L., Schmutz U., Balázs B., Trenchard L., Eyden-Wood P., Bos E., Sutton G., Blackett M., 2013, « Short food supply chains and local food systems in the EU. A state of play of their socio-economic characteristics ", JRC Scientific and Policy Reports, Joint Research Centre Institute for Prospective Technological Studies, European Commission.

Knoepfel P., Larrue C., Varone F., Savard J.-F., 2015, Analyse et pilotage des politiques publiques : France, Suisse et Canada, Presses de l'université du Québec. 423 p.

Loi $n^{\circ}$ 2010-874 du 27 juillet 2010 de modernisation de l'agriculture et de la pêche, JORF $n^{\circ} 0172$ du 28 juillet 2010 page 13925 , texte $n^{\circ} 3$

Loi $\mathrm{n}^{\circ}$ 2014-1170 du 13 octobre 2014 d'avenir pour l'agriculture, l'alimentation et la forêt, JORF $\mathrm{n}^{\circ} 0238 \mathrm{du} 14$ octobre 2014 page 16601 , texte $\mathrm{n}^{\circ} 1$.

Loi n 2015-991 du 7 août 2015 portant nouvelle organisation territoriale de la République, JORF $n^{\circ} 0182$ du 8 août 2015 page 13705 , texte $n^{\circ} 1$.

Loi $n^{\circ} 2018-938$ du 30 octobre 2018 pour l'équilibre des relations commerciales dans le secteur agricole et alimentaire et une alimentation saine, durable et accessible à tous, JORF $\mathrm{n}^{\circ} 0253 \mathrm{du} 1^{\mathrm{er}}$ novembre 2018 , texte $n^{\circ} 1$.

Maréchal G., Noël J., Wallet F., 2018, « Les projets alimentaires territoriaux (PAT) : entre rupture, transition et immobilisme?», Pour, vol. 2, n² 234-235, p. 261-270.

Moragues-Faus A., Morgan K., 2015, « Reframing the foodscape: The emergent world of urban food policy », Environment and Planning A, vol. 47, n 7, p. 1558-1573.

Muller P., 1990, Les politiques publiques, Paris, PUF, coll. « Que sais-je ?», 127 p.

Muller P., 2005, «Esquisse d'une théorie du changement dans l'action publique. Structures, acteurs et cadres cognitifs ", Revue française de science politique, vol. 55, n 1, p. 155-187, https:// doi.org/10.3917/rfsp.551.0155.

Parlement européen et Conseil, 2013, Règlement (UE) n 1305/2013 du Parlement européen et du Conseil du 17 décembre 2013 relatif au soutien au développement rural par le Fonds européen agricole pour le développement rural (Feader) et abrogeant le règlement (CE) du Conseil, JORF $n^{\circ} 1698 / 2005$.

Pham H. V., Berriet-Solliec M., 2018, « La politique de développement rural en France. Premières analyses des programmes de développement rural dans les régions françaises métropolitaines sur la période 2014-2020 », Économie rurale, n 363, p. 141-159, https://doi.org/10.4000/ economierurale. 5450

Pivot J.-M., Mondot R., 2005, « Politiques de développement rural et niveaux de décision : une approche organisationnelle », Ingénieries eau agriculture territoires, numéro spécial Feader, p 145-153. 
Praly, C., Chazoule C., Delfosse, Mundler P., 2014, « Les circuits de proximité, cadre d'analyse de la relocalisation des circuits alimentaires ", Géographie, économie, société, vol. 16, nº 4, p. 455-478, https://doi.org/10.3166/ges.16.455-478.

Réseau rural français, 2017, « La mise en œuvre de la politique européenne de développement rural 2014-2020 en France », https://www.reseaurural.fr/sites/default/files/documents/fichiers/ 2017-11/2017_feader_fiche_de_synthese_politique_developpement_rural.pdf, consulté le 18 mai 2020.

\section{NOTES}

1. Loi $n^{\circ} 2010-874$ du 27 juillet 2010 .

2. Décret $\mathrm{n}^{\circ}$ 2019-1344 du 12 décembre 2019.

3. Loi $\mathrm{n}^{\circ} 2014-1170$ du 13 octobre 2014.

4. Loi $n^{\circ} 2018-938 \mathrm{du} 30$ octobre 2018.

5. Les taux d'aide prévus peuvent être augmentés de 10 points de pourcentage supplémentaires dans le cas des opérations concernant les CACP (Parlement européen et Conseil, 2013).

6. Loi $n^{\circ} 2015-991$ du 7 août 2015 portant nouvelle organisation territoriale de la République.

7. L'ODR est un centre de ressources créé et géré par Inrae pour analyser et évaluer les politiques rurales territorialisées. Il rassemble des bases de données se rapportant aux mesures de politiques agricoles et agro-environnementales, aux activités agricoles et, plus généralement au développement rural. L'ODR est également un outil pour les travaux d'évaluation des PDR, à ce titre il est financé par le Feader.

\section{RÉSUMÉS}

Cet article analyse la place des chaînes alimentaires courtes de proximité dans la politique de développement rural (PDR) en Occitanie. L'analyse textuelle des PDR Midi-Pyrénées et Languedoc-Roussillon 2014-2020 et de leurs respectifs appels à projets, permet d'identifier les mesures d'appui aux CACP. Les résultats soulignent un soutien timide mais plus marqué en Languedoc-Roussillon. Les contours de la future PAC (baisse du budget, passage à un plan stratégique national unique...) conditionneront l'autonomie laissée aux Régions pour définir des mesures de soutien aux CACP adaptées aux exigences locales. En plus des soutiens aux investissements physiques, à l'installation et à la coopération, les formations et la mise en réseaux des acteurs du territoire devraient être appuyées.

This article analyzes the place of short local chains (SLC) in rural development policy (RDP) in Occitanie Region. We identify measures related to SLCs through a textual analysis of the MidiPyrenees and Languedoc-Roussillon 2014-2020 PDRs and their respective calls for projects. The results underline a timid support but more marked in Languedoc-Roussillon. The contours of the 
future CAP (decrease in the budget, transition to a single national strategic plan, etc.) will condition the autonomy left to the Regions to define support measures for SLCs adapted to local requirements. In addition to support for physical investments, installation and cooperation, training and networking of actors in the territory should be supported.

\section{INDEX}

Keywords : rural development policy, short local chains, proximity, CAP, Occitanie, public support

Mots-clés : politique de développement rural, circuits courts, proximité, PAC, Occitanie, soutien public

\section{AUTEURS}

\section{FREDÉRIC WALLET}

Frédéric Wallet est docteur en économie, il anime le programme PSDR depuis 2008. Ses travaux portent sur les processus d'innovation dans les régions rurales, et les initiatives en faveur de la reterritorialisation de l'alimentation, université Paris-Saclay, Inrae/AgroParisTech, UMR SADAPT frederic.wallet@agroparistech.fr $\mathrm{N}^{\circ}$ Orcid : 0000-0002-9784-2567

\section{MELISE DANTAS MACHADO BOUROULLEC}

Melise Bouroullec est docteur en ingénierie de production, option gestion des filières agroalimentaires, elle conduit des travaux qui portent sur les mécanismes de coordination des dispositifs de mise en marché collective des produits agricoles et agroalimentaires, université de Toulouse, Inrae, INP-Purpan, UMR AGIR

melise.bouroullec@purpan.fr $\mathrm{N}^{\circ}$ Orcid : 0000-0001-6265-3849 